\title{
La bande dessinée numérique : le triomphe du linéaire sur le tabulaire
}

\author{
Philippe Paolucci \\ LERASS - Université Toulouse 3
}

\section{Introduction}

À regarder de près la production scientifique sur la bande dessinée, on constate que la question de la transition numérique occupe une place de choix dans nombre de travaux actuels. Les possibilités formelles introduites par le numérique (usage de vidéos, contenus interactifs, etc.) ${ }^{1}$ ont ainsi fourni la matière à plusieurs recherches d'inspiration sémiologique (Boudissa; Rageul; Paolucci). En complément à ces réflexions d'ordre formel, des approches plus sociologiques se sont intéressées à l'émergence de nouveaux usages d'écriture et de lecture, plus précisément aux nouvelles formes d'interaction entre auteur et lecteur (Falgas). Enfin, on rappellera que l'histoire de la BD numérique — des années 1980 à nos jours — a fait l'objet d'un premier essai sous la plume de Julien Baudry. S'inscrivant dans une perspective diachronique centrée sur la matérialité et l'évolution des supports d'inscription, Baudry retrace de manière convaincante les grandes étapes qui ont ponctué l'installation du $9^{\mathrm{e}}$ art dans la culture numérique (œuvres sur CD-ROM à la fin des années 1990, numérisation des albums, essor des blogues-BD, etc.). Si ces travaux, issus de champs disciplinaires distincts (arts plastiques, information-communication, histoire), présentent certes des différences (sur les plans méthodologiques et théoriques par exemple), et si leurs conclusions peuvent diverger par endroits, un point semble faire consensus : le numérique bouleverse le secteur de la bande dessinée dans tous ses aspects, tant du côté de la production (expérimentation de formes nouvelles, redéfinition du rapport auteur/éditeur, enjeux économiques, etc.) que de la réception (nouveaux usages de lecture, etc.). En cela, l'entrée dans l'ère numérique constitue bel et bien un changement de support « bien plus radicalement nouveau que ne l'était le passage de la revue à l'album » (Robert 14). On sait par exemple que les propriétés calculatoires du support informatique ont ouvert des possibilités inédites quant à la juxtaposition séquentielle des images. À l'instar de sa devancière papier, la BD numérique repose en effet sur la mise en séquence de plusieurs vignettes. Celles-ci, cependant, ne sont plus nécessairement étalées au sein d'une même page, c'est-à-dire offertes d'emblée à la vision synoptique, mais peuvent apparaître une à une sous l'action gestuelle du lecteur. On pense notamment aux BD-diaporamas, lesquelles reposent sur un mode de lecture exclusivement basé sur le clic (à

1 On pourrait ajouter à cette liste l'usage de séquences sonores, ou encore l'utilisation d'effets de parallaxe (à l'image de la BD Hobo Lobo of Hamelin de Stefan Zivadinović: http://hobolobo.net/). 
chaque clic, une nouvelle unité narrative apparait à l'écran). De façon analogue, les bandes dessinées à défilement vertical ou horizontal impliquent un dévoilement progressif des images à mesure que l'on déroule l'ascenseur du navigateur Internet.

Le numérique confère donc à la lecture un caractère fortement linéaire. Qu'il s'agisse de presser un bouton ou de manipuler une barre de défilement, les inscriptions (bulles, vignettes, etc.) sont appelées à s'enchaîner à l'écran selon un ordre prédéterminé. Nous touchons ici à une différence essentielle entre BD papier et BD numérique. Si la première repose en grande partie sur la dimension spatiale, en ce sens que l'élaboration et l'interprétation d'une planche papier consistent respectivement à organiser (côté auteur) et parcourir (côté lecteur) l'espace fermé de la page, la seconde joue davantage sur la dimension temporelle/linéaire. C'est précisément cette primeur du linéaire sur le tabulaire que nous nous proposons d'interroger. Pour ce faire, nous articulerons notre propos en trois temps : nous reviendrons en premier lieu sur le couple tabulaire/linéaire tel qu'il s'est développé dans le champ stripologique $^{2}$ (partie 1); nous examinerons ensuite le phénomène de linéarisation induit par le numérique et ses conséquences sur la lecture. Nous verrons alors que la prévalence du linéaire a favorisé l'éclosion de deux paradigmes formels, déjà évoqués ci-dessus : d'une part les BD à défilement vertical ou horizontal, que nous désignerons sous le terme générique de «scroll-comics » $»^{3}$ (partie 2); d'autre part les BD-diaporamas (partie 3). De manière plus précise, nous montrerons que ces deux paradigmes peuvent revêtir des formes diverses selon les auteurs et les positions éditoriales. Une BD numérisée et une création expérimentale telle que Phallaina ${ }^{4}$ renvoient par exemple à deux conceptions différentes du défilement, et constituent à ce titre deux variétés bien distinctes de scroll-comics. De même, la famille des BD-diaporamas regroupe des partis-pris formels qu'il conviendra de distinguer avec rigueur (case à case, turbomédia, etc.). À partir d'une analyse d'ordre sémiologique centrée sur la distribution séquentielle des images à l'écran, nous décrirons comment chacune de ces formes ( 6 au total : 3 pour les scroll-comics, 3 pour les BD-diaporamas) privilégie le linéaire au tabulaire et redéfinit, par conséquent, les postures lecturales/interprétatives associées au $9^{\mathrm{e}}$ art. Afin de mener à bien nos analyses, nous décrirons en premier lieu les œuvres proches des planches papier (sur le plan formel) avant d'aborder

2 Dans notre propos, le terme «stripologie » désigne un « courant théorique qui envisage la bande dessinée comme une forme narrative visuelle et qui se donne pour tâche de décrire ses procédés formels. » (Morgan 11).

3 Nous empruntons cet anglicisme aux théoriciens Matteo Stefanelli et Anthony Rageul. Afin d'éviter les répétitions lexicales, nous parlerons également de « BD à défilement ».

4 Phallaina est une BD numérique réalisée par Marietta Ren, en collaboration avec France Télévision. Sur le plan formel, l'œuvre se présente comme une seule et longue bande horizontale, une sorte de frise continue que l'on déroule de la gauche vers la droite. Cette bande dessinée, originale à plus d'un titre, fera l'objet d'une description plus détaillée dans la suite de cet article. 
des formes plus spécifiquement numériques. Précisons que cet éloignement progressif vis-à-vis de la $\mathrm{BD}$ imprimée n'est pas de nature chronologique (les auteurs ne se sont pas, au fil du temps, écartés graduellement de l'héritage papier), mais constitue davantage un point de vue à partir duquel il est possible de théoriser la BD numérique. Notre intention n'est donc pas de retracer un processus évolutif allant de formes considérées comme imparfaites à des formes numériques censément plus abouties. Plutôt que diachronique, notre approche se veut synchronique : il s'agit en effet de décrire les principales tendances formelles actuellement en usage, de dégager au sein de paradigmes généraux (les scroll-comics et les BD-diaporamas) des formes diverses, appréhendées selon leur distance à l'égard d'un modèle pris en référence (ici, la planche papier).

\section{Quelques remarques sur le couple linéaire/tabulaire}

Introduite par Fresnault-Deruelle dans un article de 1976, la dualité linéaire/tabulaire a profondément infusé les études sémiologiques consacrées à la bande dessinée. Pour mémoire, cet article fondateur interrogeait le couple linéaire/tabulaire à la lumière de l'évolution des supports d'inscription. Selon Fresnault-Deruelle, la saisie linéaire du récit est ainsi privilégiée dans les strips de presse, le lecteur étant invité à suivre une suite de vignettes aux dimensions identiques. Cette propension au linéaire est intimement liée à un «impératif commercial qui exige qu'on termine chaque [bande] sur un temps fort permettant la relance diégétique et incitant le lecteur à se reporter régulièrement à son quotidien habituel» (14). Le passage du strip à la planche favorise en revanche les compositions tabulaires. Le fait d'ériger la page en «entité commerciale de narration» aiguille les auteurs vers des mises en scène « où les images, en plus de leur valeur diégétique propre, devaient s'inscrire dans une structure coiffante, à la fois seconde et esthétiquement pré-formante » (17). On le voit, le binôme linéaire/tabulaire induit une distinction entre un espace de lecture (le strip en tant que succession de cases) et un espace de vision (la planche en tant que surface au sein de laquelle les images, visibles d'un seul coup d'œil, peuvent tisser des liens translinéaires). Dans le domaine français, la fortune éditoriale de l'album (Lesage) a logiquement conduit les sémiologues à délaisser le strip de presse au profit de la «tabularité de la planche» (Hommage 124), considérée alors comme «l'unité de base de la BD»(Ibid.). Dans cette perspective, les théoriciens ont longuement insisté sur la possibilité d'embrasser du regard l'intégralité de la mise en page avant de suivre le découpage séquentiel des vignettes ${ }^{5}$. Comme le rappelle à ce sujet Thierry

5 Le linéaire relèverait donc de la temporalité de la lecture, alors que le tabulaire activerait l'idée d'une organisation spatiale offerte au regard. Cette dialectique entre le temporel et le spatial apparaît clairement dans les écrits sémiologiques. Dans son essai Système de la bande dessinée, Thierry Groensteen distingue ainsi le découpage, qui renvoie à la segmentation temporelle des cases (ce qu'il appelle la chrono-topie), et la mise en page, qui désigne la distribution des images dans l'espace de la page (ce qu'il appelle la spatio-topie). Précisons 


\section{Groensteen :}

[...] lisant une bande dessinée, nous procédons d'abord à une saisie globale de la page et même, le plus souvent, de la double page [...], et puis nous procédons à la lecture proprement dite, c'est-à-dire à un déchiffrement case à case. (L'excellence de chaque art 18)

La saisie graduelle du récit est donc constamment contrebalancée par un voir ensemble, «chaque double page [s'offrant] au regard avant d'être déchiffrée case par case » (Formes et politique 48). Cela posé, il convient de noter que la tabularité ne s'applique pas seulement à l'échelle de la planche. Toute vignette peut en effet capturer l'attention du lecteur (beauté et détail du dessin, etc.), quitte à le détourner de la continuité linéaire du récit. Ainsi que le soutient Pierre Masson:

L'opposition linéaire-tabulaire, pour séduisante qu'elle soit, est une notion assez flottante, dont on peut trouver les traces jusqu'au sein de la simple vignette, lisible à la fois comme un tableau isolé et comme un fragment d'un message complexe. (60)

Cette remarque de Masson nous invite à distinguer deux conceptions de la tabularité : selon les cas, cette dernière peut soit renvoyer au tableau pictural (image pleine page, vignette considérée isolément), soit faire référence au tableau en tant qu'intersection de lignes et de colonnes (la planche appréhendée dans sa globalité). À notre connaissance, les théoriciens de la bande dessinée n'ont jamais clairement différencié ces deux acceptions de l'adjectif «tabulaire », ce qui n'a pas échappé à Pascal Robert :

$\mathrm{La} \mathrm{BD}$ serait prise dans une tension entre récit et tableau [...]. Le tableau dont il est question est-il tendanciellement le tableau de peinture que l'on regarde avec soin ou le tableau comme technologie intellectuelle, comme diagramme, comme outil d'organisation global de la page? Il semble que Peeters [...] oscille entre les deux sans véritablement choisir. Or, il s'agit de deux outils sensiblement différents, mais que nous récusons tout autant. Car nous rejetons, nous y reviendrons, l'assimilation (avancée également par P. Fresnault-Deruelle) de la case au tableau de peinture, comme il nous semble discutable de confondre la dynamique spatiale de la composition [...] avec la tabularité d'un tableau à vocation gestionnaire ou scientifique. (La bande dessinée, une intelligence 44-45)

enfin que la notion de « tabularité » peut aussi renvoyer à la possibilité, pour tout lecteur, de parcourir un texte de façon non-linéaire, «d'accéder à des données nouvelles dans l'ordre qu'il choisit, en cernant d'emblée les sections qui l'intéressent» (Vandendorpe, 39). Par le truchement d'outils aussi bien péritextuels (table des matières, index, annexes, etc.) qu'intratextuels (scansion en chapitres, division en paragraphes, etc.), le lecteur peut circuler à sa guise dans l'œuvre, sans nécessairement suivre l'ordre imposé par la pagination. Du fait de son fonctionnement hypertextuel, la littérature numérique a mis ce rejet de la linéarité au cœur de l'expérience de lecture. Dans le domaine de la BD numérique, ces narrations hypertextuelles ne sont pas inexistantes, mais restent à ce jour cantonnées à quelques expérimentations isolées - on pense entre autres aux créations d'Anthony Rageul (http://www.anthonyrageul.net/), ou encore aux productions récemment mises en ligne par le collectif RVB (https:// collectionrvb.com/). Sur cette approche de la tabularité, voir Vandendorpe, p. 41-50. 
Pour notre propos, la notion de tabularité est intéressante en ce qu'elle active l'idée de perception synoptique, c'est-à-dire la possibilité, pour le lecteur, d'appréhender plusieurs vignettes simultanément. Nous établissons donc un rapprochement entre la planche et le tableau dans son acception scientifique (intersection de lignes et de colonnes), les deux étant semblables à des structures quadrillées appréhendables d'un seul tenant.

Toute la question est alors de savoir ce qu'il advient de cette tabularité en contexte numérique. Comme nous l'évoquions plus haut, les spécificités de la lecture sur écran ont pour conséquence de minorer la dimension tabulaire au profit d'une approche linéaire du récit ${ }^{6}$. On sait par exemple que le défilement, parfois imposé par les plateformes de publication (à l'image des blogues) ${ }^{7}$, prescrit un ordre d'apparition des images. De même, la « lecture cliquée », pour reprendre une expression d'Etienne Candel, invite le lecteur à découvrir, à chacun de ses clics, un nouvel élément diégétique. L'articulation entre le tabulaire et le linéaire, qui est toujours agissante sur support numérique, entre ainsi en contact avec des normes de lecture privilégiant un dévoilement progressif du contenu fictionnel ${ }^{8}$. C'est ce que nous allons approfondir dans ce qui suit, en focalisant notre attention sur les deux paradigmes formels identifiés en introduction : les scroll-comics et les BD-diaporamas.

\section{Les Scroll-comics ou la lecture défilante}

Dans un essai consacré à la transition numérique des médias, Dominique Cotte pointe une différence essentielle entre la page et l'écran :

\footnotetext{
6 Répétons-le : la linéarité, telle que nous l'entendons ici, renvoie à la manière dont le lecteur appréhende l'œuvre et les éléments qui la constituent (images et textes), non à l'enchaînement chronologique des actions représentées. Comme dans tout récit, l'auteur d'une BD numérique peut à tout moment casser la linéarité temporelle des événements diégétiques via différents procédés narratifs (analepse, prolepse, etc.). Cela posé, il n'en reste pas moins que les modalités interactives (clic ou scrolling) impliquent une saisie unidirectionnelle des unités narratives, d'où une impression de linéarité qui prédomine durant la lecture.

7 Nous rejoignons ici ce que Yves Jeanneret et Emmanuel Souchier appellent des architextes, soit des outils informatiques (un blog, un traitement de texte, etc.) dont la fonction première est de prescrire les usages. Le blog, en tant que « structure de production textuelle préformatée » (Desseilligny 74), respecte parfaitement cette définition: il encourage le scripteur à rédiger des billets concis, à utiliser le défilement comme principal modalité de lecture, enfin à accepter un mode d'interaction particulier (commentaires).

8 Le qualificatif « fictionnel» revêt ici une importance particulière. Lors de la visite d'une page web lambda, l'internaute est évidemment libre de cliquer où bon lui semble, de naviguer au sein du site suivant le parcours de son choix. En revanche, durant la lecture d'une BD numérique à dominante fictionnelle, la participation gestuelle de l'usager tend naturellement à suivre le chemin tracé par la diégèse (cette impression est bien sûr minorée lorsque l'auteur met en place une narration à embranchements multiples).
} 
[...] ce qui définit la page dans l'imprimé, c'est avant tout la limite physique du volume. En ce sens, le livre se rapproche de la tablette ou de la pierre gravée : les limites du matériau sont indépassables. En revanche, dans l'écran, la limite physique et la limite visuelle ne coïncident pas. (84)

Une production papier induit par conséquent un rapport de coïncidence entre le périmètre d'inscription circonscrit par le « support matériel » (la page en tant que surface géométriquement bornée) et les limites visuelles du «support formel» (l'organisation des signes graphiques dans l'espace paginal) ${ }^{9}$. On rencontre une idée similaire chez Groensteen lorsqu’il déclare que la planche "affecte en général l'aspect d'un rectangle, dont les dimensions sont à peu de chose près homothétiques à celles de la page » (Système de la bande dessinée, 38). L'un des effets du défilement est précisément de briser cette solidarité entre les frontières matérielles de la page et la mise en forme des inscriptions. Le format des images n'est plus déterminé par les parois paginales, mais peut s'étendre au-delà de la zone d'affichage délimitée par l'écran. Bref, la lecture sur écran délaisse la page, et par extension la structure feuilletée du livre, au profit d'une lecture défilante proche des volumen et rotulus anciens ${ }^{10}$. Ce phénomène, bien connu des historiens du livre (Vandendorpe), est usuellement désigné en stripologie sous l'expression «toile infinie ». Empruntée à Scott McCloud, la notion de "toile infinie » renvoie au fait que le défilement ne connait aucune limite préétablie. Notons par ailleurs que l'idée d'un déroulement potentiellement illimité apparait, sous des habillages terminologiques certes différents, dans nombre de travaux postérieurs à l'essai de McCloud. Dans sa thèse de doctorat, Magali Boudissa qualifie ainsi l'écran « d'espace élastique » (134), au sein duquel les vignettes deviennent des unités amovibles. Ces dernières n'ont plus d'emplacements stables, mais changent continuellement de place, peuvent se trouver dans la partie inférieure ou supérieure de l'écran selon la position de la barre de défilement. Dans un sens voisin, Anthony Rageul évoque un «espace dilatant» (112), à l'intérieur duquel les dimensions des vignettes peuvent déborder la superficie de la page-écran. L' «infinité » chez McCloud, l'« élasticité » chez Boudissa et la « dilatation » chez Rageul, autant de vocables décrivant la rencontre entre un médium historiquement lié à la page et au codex (la BD) et la remédiation numérique du rouleau. Dans le cadre de cet article, nous analyserons cette résurgence du volumen de la façon suivante : dans un premier temps seront étudiés les scroll-comics semblables, du point de vue formel, à une planche papier; dans un second temps, nous montrerons que le scrolling encourage chez certains auteurs un effacement

9 Nous empruntons le couple support matériel/support formel à Isabelle Klock-Fontanille. 10 Rappelons qu'on parle de volumen lorsque le rouleau est pensé pour un défilement horizontal, et de rotulus lorsque le déroulement a lieu sur l'axe vertical. Dans la littérature académique, le terme "volumen » est souvent utilisé pour désigner les rouleaux dans leur ensemble, quel que soit leur sens de lecture. Nous reprenons cet usage dans le cadre de cet article. 
du dispositif tabulaire de la planche, ce qui a pour conséquence immédiate de linéariser la lecture. En tout, cette démarche analytique mettra en exergue trois types de scroll-comics : les BD numérisées; les scroll-comics linéarisés; enfin les scroll-comics avec effet de continuité iconique.

\subsection{Les BD numérisées}

Dans les usages, l'expression «BD numérisée » désigne la simulation, sur un écran, du dispositif de la planche tel qu'il s'est matérialisé dans la presse et l'album (à l'image des BD hébergées sur la plateforme Iznéo). Parfois dépréciées pour leur manque d'originalité formelle, les BD numérisées sont également critiquées pour leur incapacité à reproduire les conditions de lecture d'une œuvre papier. Un des points les plus fréquemment relevés porte sur les difficultés à retrouver, au contact de l'écran, la relation haptique que noue le lecteur avec la tridimensionnalité du livre. La numérisation signerait ainsi la perte «du feuilletage et [de] la profondeur apportée par l'épaisseur de l'album » (Robert 19). Â cela s'ajoute le fait que certains écrans, en raison de leur taille réduite, empêchent un affichage complet de la planche, obligeant l'utilisateur à recourir au défilement pour prendre connaissance de l'intégralité des informations diégétiques. On pense entre autres aux écrans de Smartphone qui, comme l'a montré Julia Bonaccorsi, génèrent une tension entre le lisible et le visible :

L'hypercadre de la planche apparaît [...] partiellement à l'écran, et l'on peut en déduire qu'il ne peut jouer alors complètement sa fonction sémiotique de solidarité iconique. [...] Soit la planche est visible, et elle est illisible autrement que comme objet, soit elle est rendue lisible par un procédé de zoom et de déplacement dans le cadre, et elle est invisible en tant que planche. (122)

Autrement dit, soit le lecteur choisit d'afficher intégralement la planche, et en ce cas les images et indications verbales sont trop réduites pour être lues avec aisance (la planche est visible, mais non lisible); soit l'application d'un zoom permet de déchiffrer les contenus verbaux et iconiques, mais éjecte ipso facto le reste de la mise en page hors de la fenêtre de visualisation (la planche est lisible, mais non visible dans son entièreté).

Les BD numérisées cumuleraient ainsi deux défauts : d'une part, elles peinent à reproduire la matérialité feuilletée du livre, et désactivent par conséquent tous les effets de sens liés à la manipulation de l'album (par exemple repérer, au moyen d'un feuilletage rapide ou d'un bref retour en arrière, une correspondance plastique entre deux cases situées à plusieurs planches de distance); d'autre part, elles rendent malaisé le maintien d'une vision tabulaire de la planche, notamment sur les moniteurs de taille restreinte. Ces deux écueils sont évidemment liés, en ce sens que tous deux tendent, à leur manière, à privilégier le linéaire au tabulaire. La perte de l'épaisseur de l'album invite en effet le lecteur à s'approprier linéairement l'œuvre, planche par planche, ce qui limite fortement les lectures translinéaires autorisées par le codex. Quant à l'évacuation partielle du 
synoptique observée sur certains écrans, elle contribue également à valoriser la linéarité, dans la mesure où le lecteur est naturellement porté à déchiffrer la planche strip par strip, sans possibilité d'embrasser d'un seul tenant la composition d'ensemble.

Comme nous l'avons dit, la perte du synoptique affecte en premier lieu les écrans aux dimensions modestes. Cela signifie que le défilement, dans le cas des BD numérisées, est un outil de consultation exogène au geste créatif. La barre de défilement n'exerce aucune influence sur l'organisation formelle de l'œuvre, mais intervient dans un second temps pour pallier les restrictions d'affichage imposées par l'écran. Il en va tout autrement des scroll-comics analysés ci-après, lesquels reposent sur une prise en compte du défilement dans la juxtaposition séquentielle des images. Le support formel change alors de visage : il ne s'agit plus de simuler le dispositif de la planche, mais de prendre en compte les spécificités du défilement dans la disposition des unités narratives. La planche sous sa forme canonique, préservée tant bien que mal dans les BD numérisées, s'efface alors au profit d'agencements plus adaptés à une lecture défilante.

\subsection{Les scroll-comics linéarisés}

À l'inverse des BD numérisées, les scroll-comics qui suivent ont pour particularité de gommer la géométrie de la planche. Aux habituelles marques d'orthogonalité se substituent des supports formels plus aérés, caractérisés par une réduction des indices de tabularité (cadres, bordures, etc.). Le plus souvent, le fait d'estomper la composition tabulaire de la planche amène les auteurs à privilégier une disposition en colonne : l'œuvre se présente dès lors comme une superposition d'images que l'on déroule. Ce rejet de la tabularité dénote selon nous un effort d'harmonisation entre le support formel et l'interactivité défilante appelée par l'écran, comme si le fait d'allier la lecture à une activité de déroulement encourageait naturellement l'auteur à étager les vignettes (rarement plus de deux images côte à côte). S'ensuit une forte impression de linéarité : tout concourt en effet à avantager le défilement, à perturber le moins possible le recours à l'ascenseur. Pour désigner cette forme de $\mathrm{BD}$ numérique, reconnaissable à la linéarisation du support formel, nous parlerons dans ce qui suit de scroll-comics linéarisét ${ }^{11}$.

11 Cette appellation n'est pas complètement satisfaisante. En effet, tous les scroll-comics recensés dans cet article sont traversés par une même tendance à la linéarisation, certes à des degrés variables. De sorte que l'étiquette « scroll-comics linéarisé » pourrait s'appliquer à toute œuvre basée sur une logique défilante. Nous avons néanmoins choisi de garder cette dénomination générique qui, de notre point de vue, pointe de façon explicite le fait que certaines créations sont pensées, dès le départ, dans une approche linéaire/défilante (d'où l'usage du participe « linéarisé »), à l’inverse des BD numérisées qui gardent le souvenir d'une tabularité héritée de la $\mathrm{BD}$ papier. Nous verrons que la troisième catégorie de scroll-comics accentue encore davantage cette propension à la linéarisation, ce qui motivera une nouvelle proposition terminologique. 
Illustrons cela avec un exemple: les scroll-comics publiés sur la plateforme Delitoon. Entièrement pensées pour un déroulement vertical, les BD hébergées sur Delitoon prennent l'aspect d'un étagement de dessins ${ }^{12}$. Contrairement aux BD numérisées, il n'est plus question de transférer la planche à l'écran, mais de dérouler de haut en bas une colonne d'images. L'organisation verticale des vignettes invite alors le lecteur à parcourir le support formel selon un mouvement strictement descendant (figure 1). Dans ce cadre, l'activité de défilement est d'une certaine façon "fluidifiée », en ce sens qu'elle n'est plus interrompue par l'architecture quadrillée de la planche.

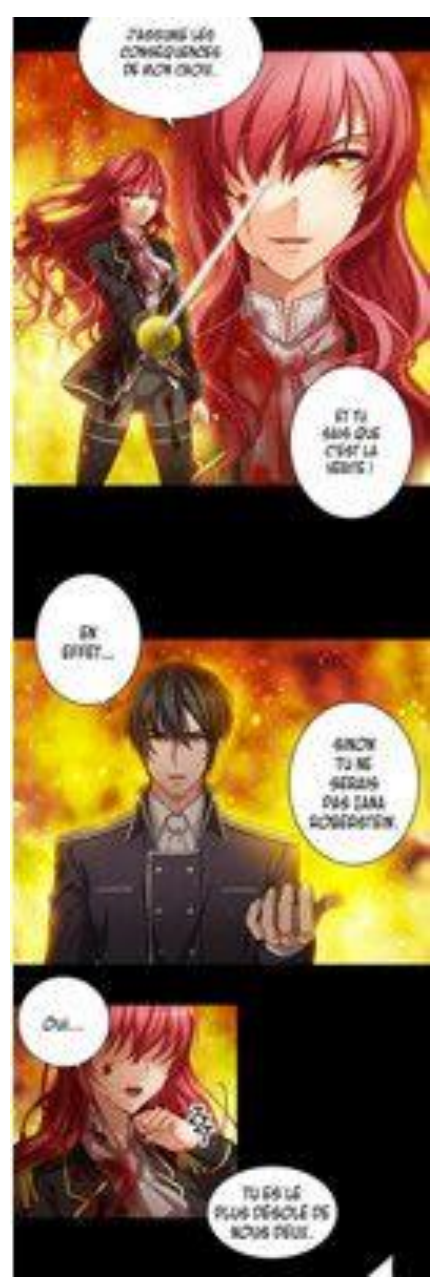

Figure 1. Exemple de scroll-comics hébergé sur Delitoon (Hyedori \& Team Adonis, Adonis, (C) Delitoon.)

Concernant la lecture, les scroll-comics linéarisés inclinent le lecteur à

12 Notons que l'objectif de Delitoon, depuis sa création, est d'importer en France le webtoon coréen, soit un modèle de BD numérique basé uniquement sur le déroulement vertical. Sur ce sujet, voir Bokyoung-Yun. 
saisir linéairement l'enchaînement des vignettes. Plus haut, nous observions que les $\mathrm{BD}$ numérisées conduisaient également à une appréhension linéaire du récit. L'expérience de la linéarité, dans les deux cas, est pourtant bien différente. Dans les BD numérisées, la linéarisation est le résultat d'un conflit entre un support formel (la planche) et un support matériel (l'écran). Sur certains moniteurs, cette situation conflictuelle empêche l'affichage complet de la planche, à moins d'en réduire considérablement la taille et d'en affecter la lisibilité. Nous l'avons vu, cette perte du synoptique tend à privilégier une lecture linéaire strip par strip. Il en va tout autrement lorsque la linéarisation du support formel est un choix volontaire, parfaitement intégré dans le processus de création. À l'inverse des BD numérisées qui, du fait de leur proximité morphologique avec les productions papier, signalent la présence d'une tabularité synoptique, les scroll-comics linéarisés sont dès le départ conçus dans une logique défilante, substituant à l'architecture quadrillée de la planche un agencement rectiligne d'images. Dans ce contexte, l'interprétation du lecteur épouse la succession syntagmatique des unités narratives, sans possibilité de quitter la route tracée en amont par l'auteur. Bref, la dimension tabulaire/synoptique de la bande dessinée n'exerce plus d'influence notable sur le processus de lecture.

\subsection{Les effets de continuité iconique}

On sait que toute bande dessinée, selon Groensteen, repose sur un principe de "solidarité iconique » (Système de la bande dessinée, 21), en vertu duquel les images, bien que séparées les unes des autres, sont toutes sémantiquement corrélées. Autrement dit, l'aspect fragmenté du support formel (la planche) n'empêche pas la construction d'une unité diégétique globale. La solidarité iconique suppose donc à la fois une discontinuité de l'expression (juxtaposition d'images distinctes) et une homogénéité sémantique du contenu (l'univers diégétique). Jusqu'à ce point, tous les scroll-comics que nous avons analysés respectent le principe énoncé par Groensteen. D'autres cependant contrarient le critère de la discontinuité sur lequel repose en partie la solidarité iconique. Certains auteurs s'attachent en effet à limiter autant que faire se peut les indices de fragmentation. Il ne s'agit pas seulement de réduire les marques de tabularité - ce que font déjà les scroll-comics linéarisés décrits plus haut —, mais d'œuvrer à une suture continue de l'espace dessiné. Le lecteur a ainsi l'impression de dérouler une seule et longue image, à la manière d'une "Tapisserie de Bayeux numérique $»^{13}$. Nous parlerons, lorsqu'un tel cas se présente, de continuité iconique.

Comme exemple éloquent de continuité iconique, on retiendra la $\mathrm{BD}$

13 Cette comparaison est convoquée à des fins exclusivement explicatives. Loin de nous l'intention d'ériger la Tapisserie de Bayeux au rang d'illustre ancêtre de la bande dessinée (sur ce point, nous nous éloignons par exemple de Scott McCloud, (op.cit). 
numérique Phallaina de Marietta Ren (figure 2). Sur le plan formel, Phallaina s'apparente à une seule et longue image au sein de laquelle la séquentialité est assurée, non par des bordures ou blancs interstitiels, mais par des changements de perspective et des effets de raccords intradiégétiques (duplication des personnages, encadrement d'une fenêtre ou d'une porte, etc.). Comme le remarque la chercheuse Hélène Crombet :

[Phallaina] fait voler en éclats le principe normatif de segmentation de la bande dessinée, donnant l'impression d'un flux continu qui s'opère dans l'illusion d'une ininterruption du parcours visuel. Cette spécificité formelle est susceptible de participer d'un mécanisme d'immersion de l'utilisateur, tout se passant comme s'il n'avait affaire qu'à une seule planche, à travers un continuum qui défait le principe d'isolement, de clôture iconique à l'œuvre traditionnellement dans la bande dessinée. (\$10)

Ainsi que le précise Marietta Ren, l'élaboration de Phallaina s'inspire « des premières formes de narration graphique comme la Tapisserie de Bayeux, les rouleaux chinois ou les fresques murales antiques $»^{14}$. C'est bien ici un idéal de linéarité qui s'exprime: l'objectif est clairement d'atténuer la tabularité du médium en effaçant les marques de séparation explicites (cadres, bordures, blancs; cf. figure 2).

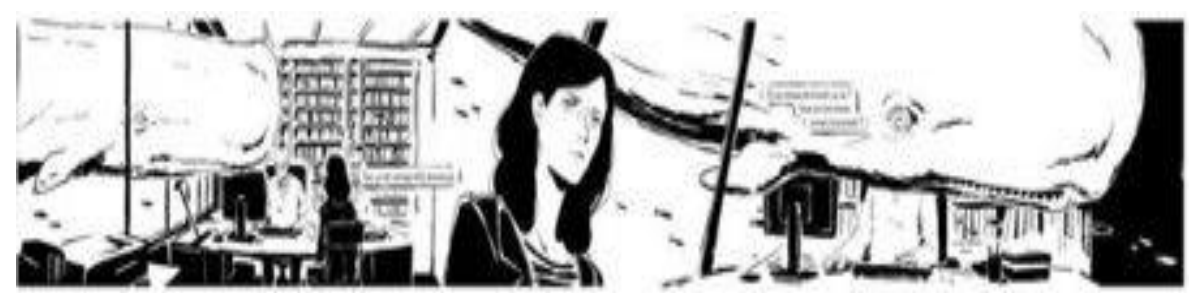

Figure 2. Marietta Ren, Phallaina, 2016, (C) Marietta REN/Smallbang/France Télévisions Nouvelles Écritures

Il est hors de doute que l'unification du décor trouve sa source dans l'usage du scrolling. Le défilement motive un espace diégétique construit sans discontinuité. Un pas de plus est franchi vers la linéarisation du support formel. L'effacement des cadres et bordures, déjà enclenché dans les scrollcomics linéarisés, atteint son faite dans les effets de continuité iconique.

En ce qui concerne la lecture, la continuité iconique conduit évidemment à une appropriation strictement linéaire de l'énoncé. L'activité lecturale consiste dès lors à appréhender les nouvelles informations apparues en bas (scrolling vertical) ou à droite (scrolling horizontal) de l'écran, informations qui seront immédiatement reliées au contenu tout juste lu. Les scroll-comics linéarisés (cf. supra) nous amenaient déjà à une conclusion analogue. La continuité iconique, en effaçant les habituelles traces de segmentation, ne fait qu'accentuer cette tendance.

14 Passage issu du dossier de presse. 


\section{Les BD-diaporamas ou la lecture par le clic}

Dans notre propos, l'appellation BD-diaporama désigne toute bande dessinée numérique dont la lecture mobilise le clic comme seule modalité interactive. Le lecteur se contente alors d'appuyer sur un périphérique externe (bouton de souris, clavier, etc.) pour faire apparaître les unités narratives à l'écran et, partant, prendre connaissance du récit ${ }^{15}$.

À l'instar des scroll-comics, nous aborderons les BD-diaporamas en nous éloignant graduellement du modèle papier. Deux grandes catégories de BDdiaporamas ont pu être ainsi identifiées. La première catégorie regroupe les créations fonctionnant sur un principe de substitution. Par substitution, nous entendons le fait de remplacer ce qui est affiché à l'écran par un nouveau contenu : à chaque clic, le strip ou la case visible à l'écran disparaît au profit d'un nouveau strip ou d'une nouvelle case. Dans notre perspective, on parlera de diaporama de strips quand la substitution est appliquée à des strips, et de case à case quand elle s'exerce à l'échelle de la vignette. Vient ensuite une seconde catégorie, constituée d'un ensemble d'œuvres reposant sur un principe de complétion. Dans ces dernières, connues sous le nom de turbomédia, le clic active l'apparition d'un nouvel élément dans la zone de représentation (une bulle, un personnage, etc.), sans nécessairement effacer les composants présents à l'écran. Il ne s'agit donc pas d'évacuer/remplacer ce qui est affiché (substitution), mais d'ajouter à chaque clic une nouvelle unité narrative dans le champ visuel, laquelle prendra place aux côtés des éléments déjà visibles. En résumé, il est question d'une complétion progressive de la zone d'affichage à mesure que le lecteur appuie sur un organe d'entrée (clavier, souris, etc.). La substitution et la complétion renvoient donc à deux manières de programmer l'apparition des inscriptions à l'écran. Dans le cadre de cet article, nous étudierons dans un premier temps les œuvres construites dans une logique substitutive (les diaporamas de strips et les case à case) avant d'aborder les créations à dominante complétive (les turbomédias).

\subsection{Les diaporamas de strips}

Commençons par les diaporamas de strips. Dans ce type de production, les strips s'affichent à l'écran selon un principe de substitution : à chaque clic, un nouveau corps d'images fait son apparition et remplace le précédent. Ajoutons que les œuvres relevant de cette catégorie peuvent prendre deux formes différentes : soit chaque page-écran donne à voir deux rangées de cases et s'apparente, grosso modo, à une planche classique coupée en son centre; soit chaque clic fait apparaittre une bande horizontale de deux, trois ou quatre cases. Ce dernier cas de figure trouve une illustration parfaite dans

15 Un tel mode de lecture rappelle évidemment les présentations PowverPoint (ou tout autre logiciel semblable), d'où l'usage du terme «diaporama», aujourd'hui largement répandu dans la littérature spécialisée. 
la BD Le ruban bleu d'Aseyn et Fibre Tigre. Dans cette création, le support formel prend l'aspect d'un petit strip composé de trois cases; le lecteur n'a alors qu'à cliquer sur la flèche orientée vers la droite, dessinée sous les images, pour afficher un nouveau triptyque (figure 3 ).
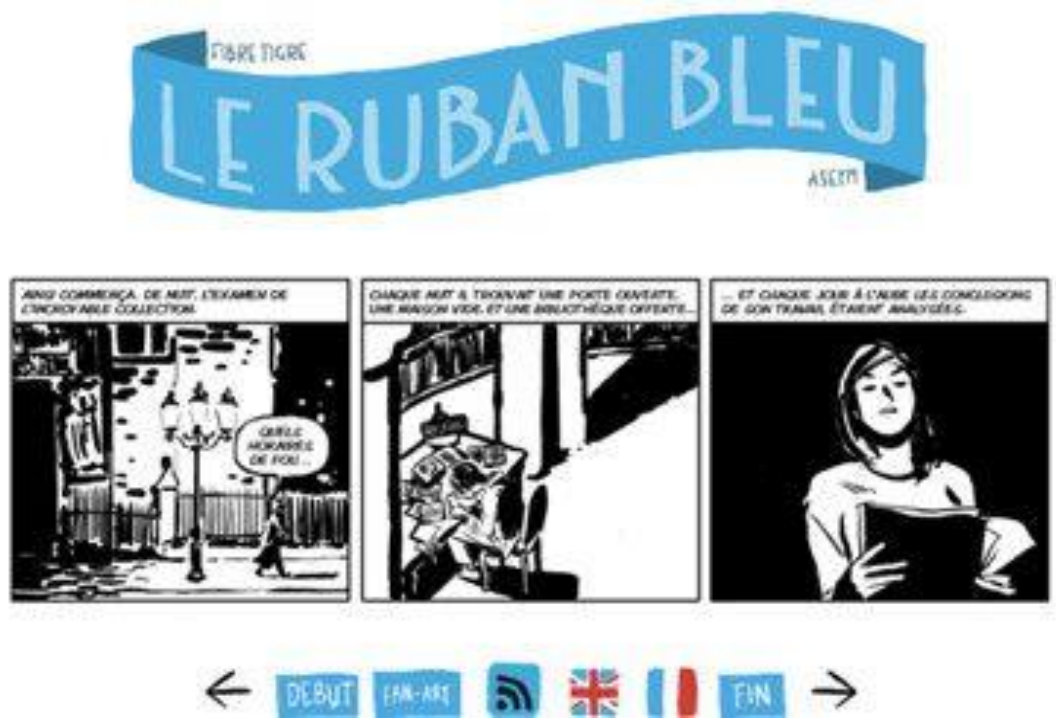

Figure 3. (C) Aseyn et Fibre Tigre, Le Ruban bleu (http://www.lerubanbleu.com/).

Comme le montre la figure 3, les diaporamas de strips renouent avec le synoptique. Le lecteur a en effet une vision complète de l'ensemble des vignettes. Ce maintien du synoptique trouve son origine dans l'utilisation du clic comme unique modalité interactive, ce qui implique une zone de représentation visible d'un seul bloc, sans nécessité de recourir au défilement. Cela entraîne évidemment une contrainte du côté de l'auteur, lequel peut difficilement cumuler plus de deux strips par écran. Au-delà de cette limite, il devient difficile d'afficher l'ensemble des vignettes sans prendre le risque de reconduire la tension entre le visible et le lisible observée dans certains scroll-comics (en particulier les BD numérisées). Rappelons brièvement comment se manifeste une telle tension : soit la planche est visible dans son entier et les informations icono-textuelles (images et bulles) deviennent illisibles, car trop rétrécies; soit les indications verbales et les dessins sont agrandis et une partie de la planche est expulsée hors écran, ce qui oblige le lecteur à réintroduire dans la zone d'affichage, via la barre de défilement, les cases non visibles. Se dessine dès lors une différence essentielle entre les BD numérisées et les diaporamas de strips : alors que les premières consistent à insérer l'intégralité d'une planche dans le cadre de l'écran, quitte à mobiliser le scrolling pour prendre connaissance de l'ensemble de la mise en page, les seconds impliquent une réduction du nombre de cases afin d'exclure toute activité défilante. Cette mise à l'écart $\mathrm{du}$ défilement explique que les BD-diaporamas étendent rarement le synoptique au-delà de deux strips. Se limiter à deux rangées d'images assure en effet une lisibilité satisfaisante sur les écrans de taille restreinte, tout en 
cantonnant la lecture à une interactivité de navigation unique : le clic. Précisons enfin que, malgré ces différences sur le plan interactif, les BD numérisées et les diaporamas de strips présentent une similitude d'ordre lectural. Dans les deux cas, le lecteur peut embrasser une tabularité synoptique, même si cette dernière est en partie amputée par les conditions d'affichage (BD numérisée) ou volontairement réduite (diaporamas de strips). S'agissant plus spécifiquement des diaporamas de strips, on notera que l'application d'un principe de substitution imprime à la lecture un caractère fortement linéaire (l'usager saisit l'œuvre strip par strip), et ce malgré la cohabitation de plusieurs vignettes dans le champ visuel.

\subsection{Les case à case}

Les case à case reposant sur la même logique substitutive que les diaporamas de strips, nous nous bornerons ici à quelques remarques complémentaires. La seule différence réside en effet dans le nombre d'unités affichées à l'écran. Comme son nom l'indique, un case à case propose au lecteur de lire chaque vignette isolément, l'une après l'autre. À chaque clic, une nouvelle image fait son apparition et se substitue à la précédente. De toute évidence, le fait de réduire l'affichage à une case unique a pour conséquence de linéariser encore davantage la lecture. Dans un diaporama de strips, la cohabitation de plusieurs cases au sein de l'écran permet de saisir plusieurs unités simultanément, partant de garder un certain contact avec les propriétés tabulaires du médium. Avec le case à case, au contraire, le regard n'appréhende qu'une image à la fois; seules restent visibles des informations contextuelles extérieures au récit (interface du navigateur web, etc.). L'attention du lecteur se porte donc exclusivement sur les relations sémantiques entre vignettes directement consécutives dans l'ordre séquentiel ${ }^{16}$.

\subsection{Le turbomédia}

À l'heure actuelle, le turbomédia ${ }^{17}$ est sans nul doute la forme de BDdiaporama la plus prisée sur le web. Comme nous l'évoquions plus haut, le turbomédia repose sur un principe de complétion : chaque clic commande l'apparition d'un nouvel élément graphique dans le champ visuel, sans

16 Dans ces conditions, la notion de tabularité pourrait être mobilisée dans son sens pictural, puisqu'il s'agit bien de contempler une vignette isolée à la façon d'un mini-tableau (cf. partie 1 sur le couple tabulaire/linéaire).

17 Notons que le vocable «turbomédia », aujourd'hui largement adopté par les acteurs du secteur (auteur, lecteur, critique, etc.), n’a jamais été clairement défini. Ce flou sémantique a sans doute favorisé l'installation de ce néologisme dans les usages, le but étant de nommer explicitement une nouvelle pratique narrative (car il faut bien lui donner un nom) sans pour autant l'enfermer dans une définition trop rigide. Pour notre part, nous retiendrons simplement que les turbomédias reposent sur un régime complétif, et constituent à ce titre une complexification des BD-diaporamas classiques. 
nécessairement effacer les informations (dessins et/ou textes) déjà affichées à l'écran. À l'inverse des BD-diaporamas plus classiques, basées sur un principe de substitution, les turbomédias invitent le lecteur à compléter progressivement, clic après clic, les éléments constitutifs d'un strip, voire d'une vignette. Il devient alors possible de recomposer petit à petit le contenu icono-textuel d'une case. Comme exemple prégnant de cette logique complétive, on pourrait citer la gestion des bulles dans nombre de turbomédias. Sur support papier, nous savons que l'emplacement des bulles respecte deux impératifs :

1. les phylactères doivent, par leur position, indiquer clairement la chronologie des répliques;

2. chaque bulle doit prendre place dans une zone faiblement sémantisée (bleu du ciel, etc.), de façon à ne pas empiéter sur les éléments diégétiques essentiels à l’intelligibilité du récit.

Nous n'avons relevé aucune entorse au second principe dans la production actuelle, et ce quelle que soit l'option formelle considérée (scrollcomics ou BD-diaporama). L'énonciation turbomédiatique, en revanche, rend le premier impératif beaucoup moins coercitif. Lorsque chaque clic appelle le surgissement d'une nouvelle bulle, l'attention du lecteur se porte inévitablement sur celle-ci, quelle que soit sa position au sein de l'image. Alors que sur papier, la succession des répliques implique une trajectoire oculaire du haut vers le bas (on lit d'abord les bulles situées vers la bordure supérieure de la case, puis l'on descend vers la bordure inférieure), rien n'empêche un auteur de turbomédia de renverser cette convention de lecture. Une première bulle peut par exemple apparaitre en bas de la vignette et la suivante dans la partie supérieure : cela ne gênera en rien le processus de lecture, dans la mesure où la succession chronologique des phylactères repose moins sur des critères spatiaux (disposition hiérarchisée au sein de la case) que temporels (le lecteur est logiquement porté à lire la dernière réplique affichée). Cette primauté du temps sur l'espace — ou du linéaire sur le tabulaire - apparait avec évidence lorsqu'on compare le turbomédia Le Sourire de Rose de Sacha Goerg à son adaptation papier, parue chez Casterman en 2014 (figure 4$)^{18}$.

18 Le Sourire de Rose est paru en plusieurs épisodes dans la revue Professeur Cyclope. À l'heure où nous écrivons ces lignes, et pour une raison que nous ignorons, la revue n'est plus accessible sur le web. 


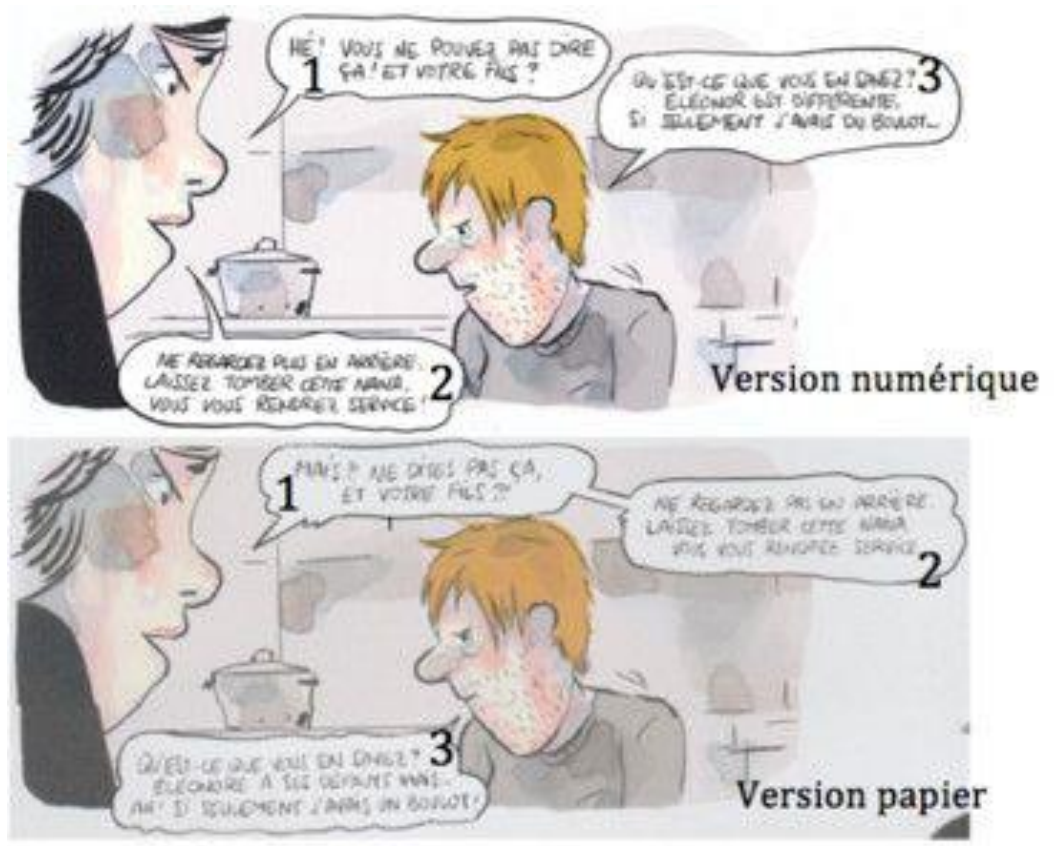

Figure 4. En haut : Sacha Goerg, Le Sourire de Rose, épisode 2, octobre 2013, (C) Professeur Cyclope/Goerg; en bas : la même scène extraite de la version papier (mai 2014, (c) Casterman). Reproduit avec l'aimable autorisation de l'auteur et des éditions Casterman.

L'extrait ci-dessus montre une discussion entre une jeune femme (la fameuse Rose) et un dénommé Desmond. La case compte au total trois bulles. Dans la version numérique, les deux premiers clics affichent chacun un phylactère associé au personnage féminin, l'un disposé en haut à gauche de la case, l'autre en bas à gauche. Un troisième clic, enfin, fait apparaittre la dernière bulle — reliée au personnage masculin — en haut à droite. Le processus de lecture se veut par conséquent parfaitement linéaire : le lecteur appréhende dans un premier temps les deux phylactères situés sur la gauche, avant de rencontrer la seule réplique énoncée par le protagoniste masculin. Dans l'adaptation papier, Goerg propose un autre agencement. Les deux bulles émises par Rose apparaissent en haut de la case et sont reliées par un appendice; Desmond, quant à lui, prend la parole en bas à gauche de la vignette. Le passage du numérique au papier oblige donc l'auteur à refaçonner le support formel. Le turbomédia reposant sur une logique complétive strictement linéaire (la succession des clics complète petit à petit le support formel), l'ordre de lecture des phylactères correspond à l'ordre d'apparition des unités à l'écran. Dans ce cadre, la dernière bulle affichée apparait d'emblée comme la prochaine unité à lire. Pour la version album, en revanche, Goerg renoue avec une disposition conforme aux routines de lecture d'un ouvrage papier. La matérialité paginale — non dynamique à l'inverse du numérique - impose une nouvelle répartition des bulles dans l'espace de la case, de façon à ne laisser aucun doute sur les tours de parole. 
Comme le montre le turbomédia de Sacha Goerg, l'application du régime complétif peut déboucher sur une mise à distance des habitudes de lecture héritées de la culture imprimée. Le Sourire de Rose n'est bien sûr pas un cas isolé; nombre de turbomédias se détachent des usages de lecture consolidés sur support papier. On ne compte plus à ce sujet les œuvres qui dérogent à l'orientation gauche-droite caractéristique de la lecture occidentale. Un clic peut par exemple faire surgir un personnage dans la partie droite d'une case, et le clic suivant signer l'apparition d'un autre protagoniste dans la partie gauche, sans que cela choque le lecteur. Là encore, le fonctionnement linéaire/complétif du turbomédia fait qu'un ordre d'apparition droitegauche, bien qu'opposé au sens de lecture habituelle, ne sera pas perçu comme perturbant.

Enfin, on notera que le turbomédia, du fait de ses propriétés complétives, introduit un nouveau rapport à la temporalité diégétique. Par ses clics successifs, le lecteur fait apparaître un à un les micro-événements contenus en chaque image (par exemple l'ordre des répliques chez Goerg), dévoile leur rapport de consécution et pénètre, ce faisant, dans ce que nous pourrions appeler la temporalité interne à la case. Chaque case est en effet porteuse d'un événement diégétique pourvu d'une temporalité propre. Or, comme le rappelle Harry Morgan, les études sémiotiques/stripologiques ont surtout étudié la construction du temps diégétique à travers le jeu des cases, sans vraiment considérer les rapports de consécution enfermés en chaque image : Il nous faut, pour commencer, rappeler la présence d'une temporalité à l'intérieur de la vignette considérée isolément. La théorie d'inspiration sémiologique appliquée aux littératures dessinées a eu tendance à se focaliser dès le début sur le système imagier (autrement dit, sur le dispositif et sur les relations entre les vignettes), de sorte que la dynamique a été examinée de façon préférentielle dans le cadre de la pluralité des cases. Mais la vignette, considérée isolément, intègre les rapports de causalité et de consécution qui définissent le récit. (308)

En déplaçant la séquentialisation du récit à l'intérieur de la vignette, le turbomédia pointe un impensé des travaux stripologiques. Par son geste, le lecteur recompose l'ensemble des actions qui, du fait de leur enchaînement, constituent la temporalité propre à la case. Autrement dit, la répétition à intervalles plus ou moins réguliers d'une action ponctuelle (le clic) facilite la mise en place d'un régime complétif et permet au lecteur d'afficher linéairement, l'un après l'autre, les divers composants d'une même vignette.

\section{Conclusion}

En guise de conclusion, nous proposons de rapprocher ci-après les deux paradigmes formels décrits dans cet article. Pour ce faire, nous avons regroupé les formes analysées en trois grandes paires, chaque paire étant constituée d'un scroll-comics et d'une BD-diaporama. 
$B D$ numérisée/diaporama de strips : dans cette première catégorie, les œuvres présentent un support formel encore très imprégné de la géométrie tabulaire des œuvres papier. On pense en premier lieu aux BD numérisées, c'est-àdire aux simulations numériques de planches. Nous l'avons vu, l'usage du scrolling dans ce type de production n'est pas systématique, mais dépend des dimensions plus ou moins généreuses de l'écran. Cela signifie que l'appartenance des $\mathrm{BD}$ numérisées à la catégorie des scroll-comics est le résultat d'un paramètre contextuel (diversité des écrans) extérieur au processus de création. Quant aux diaporamas de strip, l'exclusion du scrolling dans les modalités de lecture implique une réduction du support formel à une ou deux rangées de vignettes. Rappelons enfin que ces deux formes tendent à minorer, chacune à leur manière, le rôle du synoptique dans l'accès au sens, soit en mettant celui-ci au centre d'une tension entre le visible et le lisible (cf. la lecture d'une BD numérisée sur les moniteurs de taille modeste), soit en le réduisant à un nombre restreint de cases (diaporama de strips).

Scroll-comics linéarisé/ case à case : cette deuxième catégorie se caractérise par une linéarisation accrue du support formel et de la lecture. Concernant les scroll-comics, on note une réduction des marques de tabularité au profit d'une disposition superposée/étagée des vignettes. En ce qui concerne les BD-diaporamas, cette prévalence du linéaire s'observe en particulier dans le case à case. Le lecteur appréhende de façon isolée, sans possibilité d'embrasser du regard plusieurs unités à la fois, l'image affichée à l'écran. Qu'il s'agisse donc de scroll-comics ou de BD-diaporama, l'atténuation des traces de tabularité linéarise la lecture.

Scroll-comics avec effet de continuité iconique/turbomédia: cette troisième catégorie signe un écart important avec la BD papier. Dans les effets de continuité iconique, la linéarisation du support formel franchit une nouvelle étape. Les marques de segmentation tendent à s'effacer au profit d'une suture ininterrompue de l'espace diégétique. La séquentialisation est alors assurée par des raccords intradiégétiques (duplication des personnages, etc.). La continuité iconique est donc le résultat d'une adéquation maximale entre l'activité de défilement et l'élaboration du support formel. De son côté, le turbomédia attire l'attention du lecteur sur la « séquentialisation [...] de la case elle-même » (Bonaccorsi 119). Par le biais du régime de la complétion, l'énonciation turbomédiatique invite le lecteur à recomposer les événements diégétiques constitutifs de la vignette.

Évidemment, nous n'ignorons pas que les formes décrites dans cette contribution sont loin d'englober l'ensemble de la production actuelle. Les créations d'Anthony Rageul, par exemple, peinent à trouver une place dans 
nos descriptions formelles ${ }^{19}$. Dans ses réalisations numériques pour le moins atypiques, Rageul multiplie les dispositifs interactifs : récit bifurquant (Les Monstres d'Amphitrite), vignettes à assembler à la façon d'un puzzle (Prise de tête), modification des images via un simple survol du curseur (Romuald et le tortionnaire). Un tel usage de l'interactivité insuffle à la lecture un caractère ludique assez désorientant, le lecteur ne sachant pas avec précision quelle zone de l'écran survoler ou activer. Bref, l'usager tâtonne, teste à l'aveugle les mécanismes interactifs élaborés par l'auteur, parfois au risque de reléguer au second plan la narration et l'histoire. Cette dimension ludique n'est évidemment pas propre à Rageul. On l'observe également dans la bande dessinée Nawls de Stuart Campbell, dans laquelle le lecteur est invité à déplacer le pointeur de la souris afin d'identifier des zones cliquables, plus ou moins camouflées, qui lui permettront d'avancer dans l'histoire. On le voit, les auvres de Rageul et de Campbell témoignent d'une certaine propension au ludique. C'est bien ici la frontière avec le jeu vidéo qui est interrogée. Le numérique, support manipulatoire et interactif par essence, constitue en effet un environnement technologique «ludogène»(Vial), propice à l'émergence de contenus jouables (ou perçus comme tels). Pour cette raison, l'analyse de telles productions nécessite selon nous un dialogue entre la stripologie et les études menées au sein des game studies sur les " phénomènes de ludicisation du numérique » (Genvo et Solinski 9), lesquels phénomènes «incitent $[. .$.$] à se demander ce qui fait qu'un objet, qu'une$ interface, qu'un dispositif numérique peut être jouable, peut être adapté à une utilisation ludique ou peut potentiellement être employé/considéré/reconnu comme un jeu?» $(9-10)^{20}$. Sur ce point, il convient de noter que la tabularité de la bande dessinée peut devenir, dans certaines productions, un espace de jeu. Citons à ce sujet, outre les créations d'Anthony Rageul (cf. supra), la BD numérique Murat. Réalisée par un collectif d'auteurs tchèques, cette $\mathrm{BD}$ invite le lecteur à trouver l'emplacement de chaque vignette dans l'espace de la planche. Dans le même esprit, l'application pour Smartphone Framed propose à l'usager de tester plusieurs agencements de vignettes afin de permettre au personnage d'éviter certains pièges. Point intéressant : alors que Framed est reconnu comme un jeu vidéo (par la presse spécialisée et les joueurs), Murat est davantage associé au monde de la bande dessinée. Les deux productions, pourtant, s'appuient sur des mécanismes interactifs tout à fait semblables. À la suite de Sebastien Genvo, on expliquera ces différences de classification en rappelant qu'une expérience vidéoludique convie le joueur à faire l'exercice des possibles, à explorer les différentes stratégies qui le mèneront à la victoire, quitte à se tromper et perdre la partie. Cet exercice des possibles, couplé à l'éventualité d'un échec, est ce qui distingue un jeu vidéo d'une BD numérique. Même

19 Toutes ces réalisations sont consultables sur le site de l'auteur : http://www.anthonyrageul.net/.

20 Précisons que la nécessité d'un rapprochement entre la stripologie et les game studies apparaît également dans la thèse de doctorat d'Anthony Rageul. 
lorsqu'une bande dessinée se pare des atours du jeu, il est rare que le lecteur soit sous la menace d'un game over. Comme le dit avec justesse Elsa Caboche : «Il n'est pas (encore) possible de perdre dans une bande dessinée numérique » (152).

Fresnault-Deruelle l'observait déjà en 1976: la dialectique linéaire/tabulaire n'est pas figée, mais s'adapte à l'évolution des supports d'inscription. Le numérique ne déroge pas à la règle : malgré une primauté accordée au linéaire dans une large part de la production, certains auteurs continuent à interroger les ressources tabulaires du médium, ce qui motive un brassage intermédial avec le jeu vidéo. À ce titre, la tabularité constitue, à l'heure du numérique, une notion carrefour entre la stripologie et la ludologie. 


\section{Bibliographie}

Baetens, Jan. Formes et politique de la bande dessinée. Vrin, 1998.

---. «Hommage à Pierre Fresnault-Deruelle : Pour relire "Du linéaire au tabulaire" ", Cahiers du GRIT, no. 1, 2011, pp. 122-129.

Baudry, Julien. Cases-pixels : une histoire de la BD numérique en France. Presses Universitaires François Rabelais, 2018.

Bonaccorsi, Julia. «La bande dessinée aux prises avec la "machinerie éditoriale" du Smartphone ». Communication et langages, no. 167, 2011, pp. 87-105.

Bokyoung, Yun. «La bande dessinée numérique française et le webtoon coréen : deux modalités de la lecture sur écran ». Newvième art 2.0, 2013, http://neuviemeart.citebd.org/spip.php?article579, consulté le 25 mars 2020.

Boudissa, Magali. La bande dessinée entre la page et l'écran :étude critique des enjeux théoriques liés au renouvellement du langage bédéique sous influence numérique. 2010. Université Paris VIII, PhD dissertation.

Caboche, Elsa. « Narration numérique en images : modes d'expression, spécificité, expérimentations ». L'Engendrement des images en bande dessinée, Presses universitaires François Rabelais, 2014, pp. 143-155.

Candel, Etienne. « Entre idéaux de rupture et de continuité ». Hermès, no. 54, 2009, pp. 125-126.

Cotte, Dominique. Emergences et transformation des formes médiatiques. HermèsLavoisier, 2011.

Crombet, Hélène. «Phallaina, un voyage hallucinatoire à travers une bande défilée numérique ». Revue Française des Sciences de l'Information et de la Communication, no. 12, 2018, https://journals.openedition.org/rfsic/3397, consulté le 20 mars 2020.

Desseilligny, Oriane. «Le blog intime au croisement des genres de l'écriture de soi ». Les blogs : écritures d'un nowveau genre, L'Harmattan, 2010, pp. 73-82.

Falgas, Julien. Raconter à l'ère numérique : auteurs et lecteurs héritiers de la bande dessinée face aux nouveaux dispositifs de publication. 2014. Université de Lorraine, $\mathrm{PhD}$ dissertation.

Fresnault-Deruelle, Pierre. « Du linéaire au tabulaire ». Communication, Vol. 24, no. 1, 1976, pp. 7-23.

Jeanneret, Yves and Souchier, Emmanuel. «L'énonciation éditoriale dans les écrits d'écran ». Communication et langages, no.145, 2005, pp. 3-15.

Genvo, Sebastien and Solinski, Boris. «Introduction au numéro thématique Questionner le jouable? ». Interfaces Numériques, Vol. 4, no. 1, 2015, pp. 9-16

Groensteen, Thierry. Système de la bande dessinée. PUF, 1999.

---. L'Excellence de chaque art. Presses Universitaires François Rabelais, 2018.

Klock-Fontanille, Isabelle. «Penser l'écriture : corps, supports et pratiques ». Communication et langages, no.182, 2014, pp. 29-43. 
Lesage, Sylvain. Publier la bande dessinée : les éditeurs franco-belges et l'album, 1950-1990. Presses de l'ENSSIB, 2018.

Masson, Pierre. Lire la bande dessinée. Presses Universitaires de Lyon, 1985.

McCloud, Scott. Reinventing Comics: How Imagination and Technology Are Revolutionizing an Art Form. Perennial, 2000.

Morgan, Harry. Formes et mythopoeia dans les littératures dessinées. 2008. Université Paris Diderot, PhD dissertation.

Paolucci, Philippe. La bande dessinée numérique : entre rupture et continuité. Pour une approche socio-sémiotique de la transition numérique du $9^{\circ}$ art: l'exemple de la revue en ligne Professeur Cyclope. 2016. Université Lyon 2 Lumière, $\mathrm{PhD}$ dissertation.

Rageul, Anthony. La bande dessinée saisie par le numérique : formes et enjeux du récit reconfiguré par l'interactivité. 2014. Université Rennes 2, PhD dissertation.

Robert, Pascal. «La bande dessinée numérique en questions ». Bande dessinée et numérique, CNRS Éditions, 2016, pp. 9-29.

Robert, Pascal. La bande dessinée : une intelligence subversive. Presses de l'ENSSIB, 2018.

Stefanelli, Matteo. «Un siècle de recherches sur la bande dessinée ». La bande dessinée : une médiaculture, Armand Colin, 2012, pp. 17-49.

Vandendorpe, Christian. Du papyrus à l'bypertexte. Essai sur les mutations du texte et de la lecture. La Découverte, 1999.

Vial, Stéphane. L'être et l'écran, comment le numérique change la perception. PUF, 2013. 